\title{
Virtual Reality Programs Applications in Healthcare
}

\section{Hassan A. Aziz*}

College of Arts and Sciences, Qatar University, Qatar

\begin{abstract}
Current Virtual Reality (VR) applications in healthcare demonstrate potential abilities to address cognitive, psychological, motor, functional impairments and opportunities for training and education of clinical practitioners. Bearing in mind the overall wellness of their communities, healthcare officials had supported the idea of incorporating modern technology by increasing the budget shares and arranging for an access to advanced equipment and professional expertise. Clinicians are becoming more interested in applying VR simulation into their research and clinical trials because of the encouraging feedback published in the medical literature across a wide range of clinical health conditions. Numerous published articles propose novel concepts on applications VR technologies and their potential on disease prevention and management. Finally, the ability of sharing data collected by VR simulation systems through communication networks and electronic health records make it more attractive for the reason that it plays a role in decision making for specific case studies and distance learning.
\end{abstract}

\section{Keywords: Healthcare; Psychological; Technologies}

\section{Introduction}

Healthcare systems generate enormous and constant amounts of knowledge and information unlike 30 years ago, thus presenting a challenge to healthcare professionals to adapt to such information brokerage. To [1] overcome such challenges, advanced technological innovations offer tools to aid in healthcare professionals' education and training. One of the promising tools is Virtual Reality (VR) Training where healthcare is considered one of the biggest adopters. VR can provide a rich, interactive, engaging educational context, thus supporting experiential learning-by-doing. Providing this learning environment may raise interest and motivation of trainees and efficiently supports skills acquisitions and transfer because the learning process is established within an experimental structure. Applications of virtual training in healthcare at present vary in both the multimedia sophistication and the types of skills educated, ranging from tele-surgical applications to interactive simulations of human body and brain, to virtual worlds for emergency medicine training. Other interesting applications include the development of immersive $3 \mathrm{D}$ environments for training psychiatrists and psychologists in the treatment of mental disorders. The objective of this paper is to review the use of VR in various clinical and medical settings and procedures. Overall, the paper summarizes the current state of knowledge of VR simulation in healthcare. It creates an understanding of the topic for the reader by discussing the findings presented in recent research papers.

\section{Virtual Reality (VR) defined}

The term VR is a three-dimensional computer-generated world that can be explored interactively through a variety of computer peripheral devices. VR can [2] be any system that aims to allow a user to feel an experience through the use of special perception-changing tools. It is an illusion of reality, one that exists inside a virtual environment so that the image changes continuously depending upon the orientation and gaze of the user. Thus, users may navigate through the virtual world as though they are a part of it and will have a high level of interactivity experiencing it firsthand expressing what's known as "immersion" or "presence" [3,4].

\section{Virtual Reality in healthcare}

Military and the entertainmentindustrywere the forefront developers and users of VR, however recent medical and scientific advances made
VR technologies applicable to other fields. The VR applications wave in medicine started in 1993 were it was applied to treat mental health disorders. Earlier, VR [5-8] cognitive behavioral therapy (CBT) was employed to treat certain conditions of phobia such as height phobia. It was considered a first choice treatment since it recorded above $90 \%$ success rate. Today, VR [9-11] applications in advanced healthcare have been towards surgical procedures. Remote surgery can be performed effectively nowhere near the patient while the simulated procedure in the virtual environment is conducted by a surgeon is transmitted to a robotic instrument that mimics the actions. Other applications are [12] medical therapy, preventive medicine, visualization of databases, skill enhancement and rehabilitation, and medical education and training. In addition, VR is effective in psychotherapy to distract patients during painful procedures or to provide treatment for a wider range for anxiety disorders such as posttraumatic stress disorders [13].

Advancement in technology allowed VR systems to run on personal computers thus decreasing the implementation cost for a sophisticated system. Furthermore, with the ability to introduce digitized images to a VR system, it is possible to replicate actual places and consequently increase the possibility to treat mental health disorders (i.e. fear of public speak and social phobia). Importing photographs of friends, families and co-workers can allow patients to interact with these entities in the safety of a virtual environment before attempting the interaction in the real world $[14,2]$.

\section{Virtual Reality Exposure Therapy (VRET)}

Patients with excessive fear and anxiety emotional experiences as well as panic disorders and posttraumatic stress disorders (PTSD) may have a significant impairment in normal life functions. In recent years, Virtual Reality Exposure Therapy (VRET) was an effective novel tool for alternative treatment of anxiety disorders and specific

*Corresponding author: Hassan A. Aziz, College of Arts and Sciences, Qatar University, P.O. Box 2713, Doha, Qatar, Tel: +974-4403-4783; E-mail: Hassan.Aziz@qu.edu.qa

Received February 15, 2018; Accepted February 23, 2018; Published February 26, 2018

Citation: Aziz HA (2018) Virtual Reality Programs Applications in Healthcare. J Health Med Informat 9: 305. doi: 10.4172/2157-7420.1000305

Copyright: () 2018 Aziz HA. This is an open-access article distributed under the terms of the Creative Commons Attribution License, which permits unrestricted use, distribution, and reproduction in any medium, provided the original author and source are credited. 
phobias. Clinicians were able to use VR technology without concerns of excessive cost, loss of confidentiality and limited safety found in real world exposure. In VRET, patients are immersed within a computergenerated simulation or a virtual environment where they are exposed to specific fear stimuli. Data derived from 21 studies including about 300 subjects of VRET assessment suggested that VRET has a good potential treatment approach for several specific phobias indicated by better outcomes compared to imagine exposure where patients had to imagine feared stimulus which could be a barrier to those who lack the visualization skills. Moreover, VRET introduced an environment where patients exercised control over their own treatments that increasing their level of self-efficacy and thus gaining control over their own lives. (Parsons \& Rizzo, 2008) In another randomized trial of 97 subjects comparing VR exposure therapy to in vivo exposure for social anxiety disorder, VRET was effective for treating social fears, and improvement was maintained for 1 year [15].

\section{Virtual Reality Distraction Therapy (VRDT)}

Patients may go through pain and discomfort during many medical procedures. Traditionally, medicinal drugs are administered to ease pain. In current pain control practices, the science of human mind with various psychological techniques including distraction using virtual environment is being employed through which the patient's attention is diverted from the harmful stimulus affecting the perception of pain. Various visual and auditory senses, active emotional involvement and patient participation serve as distractors for the patient from the signals of harmful stimulus. Virtual Reality Distraction Therapy (VRDT) provides many levels of interactions to patients allowing the use of many senses thus encouraging them to be immersed in the virtual world experience. The higher the user's immersion means more attention in the virtual world and less attention to other signals of pain. A research study using VR as a distraction intervention was conducted in 2 sessions over a period of 8 weeks with 28 participants (age ranging 18-23). In each weekly session, the participants were divided into 2 groups; baseline (control) condition and VR distraction condition. The procedure involved immersing the participant's non-dominating hand up to the wrist in a cold-pressor bath; the baseline had no distraction and the VR condition (using the dominating hand) had a Playstation-2 controller as a distraction. Both groups were asked to remove their hands when the pain became unbearable. Times were measured with a stopwatch. Data showed increased pain tolerance, pain threshold and decreased pain intensity, anxiety and time spent thinking about pain in the VR distraction condition compared to the baseline (control) condition that had no distraction intervention [10-14,16].

Cancer patients experience anxiety-filled and unpleasant moments over the course of treatment. To alleviate some of this stress, hospitals use VRDT to provide a therapeutic degree of relaxation to its chemotherapy patients [17]. Another study provided encouraging support for the use of VRDT as a technique for controlling fear and anxiety during dental procedures [14].

\section{VR in cognitive and physical rehabilitation}

The application of VR is extended into cognitive rehabilitation to treat patients with brain damage and physical rehabilitation to treat stroke, acquired brain damage, Parkinson's disease and many more motor related diseases. The effectiveness of VR applications may be attributed to the interactive experimental medium where it provides a unique setting for patients to explore and act without feeling threatened. Unlike human trainers; VR provides consistency and ease of manipulation. Another advantage of VR application is the possibility for tele-rehabilitation where the services can be delivered at the patient's home via the internet.

Broeren and colleagues studied the effect of tele-rehabilitation on 29 stroke patients, aged 45-85 years [18]. The subjects were divided into three groups. The first group underwent in- or out-patient rehabilitation department in a hospital, the second group underwent rehabilitation in a facility community dwelling persons with stroke and the third group served as a reference (self-perceived health). All subjects underwent a VR environment generated by a computer were asked to move objects created in the virtual world and every time a hand activity was run. The hand movements were collected and analyzed. VR motor rehabilitation application used the haptic technology (mechanical arm) that allowed the users to manipulate virtual objects using a "data glove" where both tracking and movement's sensors were embedded in the material. Results from pre and post testing exhibited a higher median in average velocity and a decrease in median for time and hand-path ratio (HPR) parameters as compared with baseline. Subjects who underwent rehabilitation in a non-hospital environment had shown excellent adherence to the rehabilitation program thus resulting in an increased efficiency while maintaining high quality service [18].

Pilot studies showed promising results to reduce tobacco addiction with the application of the VR affordable system; eMagin HDM. Results illustrated a statistically significant reduction in nicotine addiction, abstinence rate and drop-out rate. Furthermore, knowing the benefits of VR application in medical field; diabetes technology society clinicians recently published many articles proposing studies and ideas of VR applications and their potential in obesity prevention and management [19].

The recent literature assessing the impact of VR interventions in cognitive and physical impairments elicited positive effects in patients with brain injury. Thus, VR can be considered a new and effective tool for rehabilitation after brain injury and they show a number of advantages, e.g. cost-effectiveness, compared to other interventions [20]. Despite evident progress in VR-based cognitive rehabilitation systems, there is still a need of more clinical trials for its validation.

\section{VR in surgical planning and performance}

The US Food and Drug Administration approved robotically assisted surgical devices for human surgery in 2000 and since then the number of surgeries performed by this procedure has risen. Several VR simulators are now commercially available for educational and training purposes. Surgeons can perform remote tele-surgery, minimal invasive surgery, surgical pre-planning, surgical rehearsal, image guided surgery and surgery simulation using surgery console. VR surgical simulation had been developed to perform Minimal Invasive Surgery (MIS) partly because it requires a surgeon looking at a monitor. MIS involves performing operations through minimal incisions where the surgeon can control mechanical arms, Haptic Technology, of the MIS robot while sitting comfortably in a surgical operating console $[3,4]$.

Beyond its early stages, VR simulation is integrated in many MIS training programs throughout the world. A VR surgical planning device takes actual physical data from an individual patient and combines it with computer-generated graphical data mimicking the patient's anatomy. This allows the simulation device to plan and rehearse a surgical procedure - both for training and advanced planning of an operation. The benefits of this practice include lower infection rates, less pain, reduced morbidity, fast recovery and reduced surgical errors as compared to conventional surgical procedures [21]. 


\section{VR as a diagnostic tool}

With the ability to measure and record all behavioral responses triggered by the creation of dynamic, interactive and stimulus environments; clinicians are offered with assessment and intervention options that are not obtainable by traditional methods. Technological advancement in diagnostic imaging are far ahead developed compared to other medical specialties. Radiological cross-sections are used to produce a simulated three-dimensional reconstruction of organs have become an important diagnostic tool providing clinicians with a more naturalistic view of patient's anatomy. High-resolution data [22] generation and collection are made possible with the latest systems that are easily navigated through by the addition of sophisticated visualization software to the existing hardware base thus providing a value by reducing personnel time and cost and improving clinical efficacy. The imaging modalities that represent computer-aided applications of VR in radiology include CT, MRI, x-ray imaging NM, ultrasound and computed radiography. VR simulation is also useful for autopsy, gross and microscopic examination, and ideally suited for digital pathology. Unique applications include remote supervision and annotation, 3D image viewing and manipulation, tele-pathology in a mixed-reality environment, and real-time pathology-radiology correlation [7].

\section{Limitations of VR use in the healthcare settings}

VR use is not always capable of simulating real-life situations. Purchase of the technology can be very expensive and require ongoing updates and maintenance. Similarly, the outcomes of the exercise are only as effective as the actual training provided and the person running the simulation. Training on how to use the software and/or hardware of the simulator takes up time and costs money. The trainees need to be clinically competent and able to understand the aspects of the technical accuracy in order to provide meaningful feedback. Participants, on the other hand, may resist full engagement with the technology, thus, minimizing the potential benefits of the treatment protocol [23].

\section{Conclusion}

Access to cost effective equipment, therapies and partnerships to promote state of the art treatment to patients anytime and anywhere are becoming more possible to all healthcare systems in order to develop and maintain sustainable and efficient services and quality of care. VR is now being used to provide a broad range of training to medical care professionals in anatomy instructions and surgery simulations that can simulate and control different situations that are difficult to duplicate in real life. Reduced physicians errors, cost of training resources, physician's time and increased medical specialist's proficiency and thus decreased number of malpractice are benefits of implementing of VR simulation [24].

\section{References}

1. Aziz H (2015) Health Informatics. Clin Lab Sci 28: 238-239.

2. Gega $L$ (2017) The virtues of virtual reality in exposure therapy. Br J Psychiatry 210: $245-246$.
3. Hanna MG, Ahmed I, Nine J, Prajapati S, Pantanowitz L, et al. (2003) Virtual Reality Training for Healthcare Professionals. Cyberpsychol Behav 6: 389-394.

4. Li A, Montano Z, Chen VJ, Gold JI (2011) Virtual reality and pain management: current trends and future directions. Pain Manag 1: 147-157.

5. Parsons TD, Rizzo AA (2008) Affective outcomes of virtual reality exposure therapy for anxiety and specific phobias: a meta-analysis. J Behav Ther Exp Psychiatry 39: 250-261.

6. Persky S (2011) Application of virtual reality methods to obesity prevention and management research. J Diabetes Sci Technol 5: 333-339.

7. Pourmand A, Davis S, Lee D, Barber S, Sikka N (2017) Emerging Utility of Virtual Reality as a Multidisciplinary Tool in Clinical Medicine. Games Health J 6: 263-270.

8. Rizzo A, Koenig ST (2017) Is clinical virtual reality ready for primetime? Neuropsychology 31: 877-899.

9. http://www.iactor.eu/downloads/WP\%20The\%20Potential\%20for\%20VR\%20 to $\% 20$ Improve $\% 20$ Healthcare.pdf

10. Weiderhold MD, Weiderhold BK (2007) Virtual Reality and Interactive Simulation for Pain Distraction. Pain Medicine 1: 182-188.

11. http://www.iactor.eu/downloads/WP\%20The\%20Potential\%20for\%20VR\%20 to $\% 20$ Improve $\% 20$ Healthcare.pdf

12. Aim F, Lonjon G, Hannouche D, Nizard R (2016) Effectiveness of virtual reality training in orthopaedic surgery. Arthroscopy 32: 224-232.

13. Valmaggia LR, Latif L, Kempton MJ, Ruscalafell M (2016) Virtual reality in the psychological treatment for mental health problems: an systematic review of recent evidence. Psychiatry Res. 236: 189-195.

14. Wiederhold MD, Gao K, Wiederhold BK (2014) Clinical Use of Virtual Reality Distraction System to Reduce Anxiety and Pain in Dental Procedures. Cyberpsychol Behav Soc Netw 17: 359-365.

15. Anderson PL, Price M, Edwards SM, Obasaju MA, Schmertz SK, et al. (2013) Virtual reality exposure therapy for social anxiety disorder: a randomized controlled trial. J Consult Clin Psychol 81: 751-60.

16. Rutter CE, Dahhlquist LM, Weiss KE (2008) Sustained Effaiccy of Virtual Reality Distarction. J Pain 10: 391-397.

17. Schneider SM, Hood LE (2007) Virtual Reality: A Distraction Intervention for Chemotherapy. Oncol Nurs Forum 34: 39-46.

18. Broeren J, Bjorkdahl A (2008) Virtual Rehabilitation after Stroke. Stud Health Technol Inform 136: 77-82.

19. Girard B, Turcotte V (2009) Crushing Virtual Cigarettes Reduces Tobacco Addiction and Treatment Discontinuation. Cyberpsychol Behav 12: 477-483.

20. Shin HH, Kim KM (2015) Virtual reality for cognitive rehabilitation after brain injury: a systematic review. J Phys Ther Sci 27: 2999-3002.

21. Mejiden OA, Schijven MP (2009) The value of haptic feedback in conventional and robotic assisted minimal invasive surgery and virtual reality training: a current review. Surg Endosc 23: 1180-1190

22. Ojha U, Mohammed R, Vivekanantham S (2017) Should there be greater exposure to interventional radiology in the undergraduate curriculum? Adv Med Educ Pract 8: 791-795.

23. Vaughan N, Dubey VN, Wainwright TW, Middleton RG (2016) A review of virtua reality based training simulators for orthopaedic surgery. Med Eng Phys 38 : 59-71.

24. Hanna MG, Ahmed I, Nine J, Prajapati S, Pantanowitz L (2018) Augmented Reality Technology Using Microsoft HoloLens in Anatomic Pathology. Arch Pathol Lab Med 1: 1-10. 